
\title{
25 Research Soure \\ Pre-implementation Assessment of Tobacco Cessation Interventions in Substance Use Disorder Residential Programs in California
}

\section{J. Konadu Fokuo ( $\nabla$ jkfokuo@uic.edu )}

University of Illinois at Chicago https://orcid.org/0000-0003-4299-689X

Caravella L. McCuistian

University of California San Francisco

Carmen L. Masson

Zuckerberg San Francisco General Hospital and Trauma Center

Valerie A. Gruber

Zuckerberg San Francisco General Hospital and Trauma Center

\section{Elana Straus}

UC San Francisco: University of California San Francisco

Jessie Wong

University of California San Francisco

Joseph R. Guydish

UC San Francisco: University of California San Francisco

\section{Research}

Keywords: policy implementation, Substance use treatment, Health disparities, smoking cessation

Posted Date: July 6th, 2021

DOI: https://doi.org/10.21203/rs.3.rs-519609/v2

License: (c) (1) This work is licensed under a Creative Commons Attribution 4.0 International License. Read Full License 


\section{Abstract}

Background: Across the United States, substance use disorder (SUD) treatment programs vary in terms of their tobacco-related policies and cessation services offered to clients. Guided by the Consolidated Framework for Implementation Research (CFIR), the current study identified key factors that may influence the implementation of tobacco related cessation policies and services in residential SUD programs.

Methods: We conducted semi-structured qualitative interviews with sixteen residential treatment program directors in California. The analysis was guided by a deductive approach using CFIR domains and constructs to develop codes and identify themes. ATLAS.ti software was used to facilitate thematic analysis of interview transcripts.

Findings: Guided by the CFIR constructs, themes that arose as facilitators for implementation included the relative advantage of the intervention compared with current practice, external policies/incentives to support tobacco related policy, program directors strong commitment and high self-efficacy to incorporate cessation into SUD treatment, and recognizing the importance of planning and engaging opinion leaders. Potential barriers included the SUD recovery culture, low stakeholder engagement, organizational culture, lack of workforce expertise and, lack of reimbursement for smoking cessation services.

Conclusion: The CFIR provided a valuable framework for evaluating factors that may influence implementation of tobacco policies and services in SUD treatment. In order to support successful implementation, residential SUD programs (staff and clients) require extensive education on the effectiveness of tobacco cessation efforts on health outcomes and publicly funded SUD treatment programs should receive support through expanded reimbursement for tobacco cessation services.

\section{Background}

Smoking prevalence remains disproportionately high among populations affected by substance use disorders (SUDs).(1) Among people in SUD treatment programs the smoking prevalence is 2 to 4 times higher than the general population.(2) Those seeking SUD treatment also experience greater smokingrelated health disparities as compared with the general population.(3) Given the high prevalence of smoking and the deleterious effect that tobacco can have on long term substance use, $(4,5)$ integration of smoking cessation services into SUD treatment is needed.

Research from several SUD treatment programs highlight the potential benefits of integrating tobaccorelated services and tobacco-free grounds policies into SUD treatment.(6-9) States such as New York, New Jersey, Oregon, Utah, and Texas have introduced statewide policies that support the integration of smoking cessation services into SUD treatment.(10-14) In California, a branch of the department of public health, the California Tobacco Control Program (CTCP) has supported tobacco cessation among people with SUD by offering grant funding to treatment programs to implement tobacco free policies.(15) 
Despite the efforts of these programs, challenges remain that can influence successful integration of smoking cessation services.

A 2017 systematic review examined the barriers and facilitators to smoking cessation for people in SUD treatment.(16) Results from the systematic review suggest that many persons with SUDs were motivated toward smoking cessation but were not offered support. Some people with SUD felt interventions should be delivered subsequent to SUD treatment; while others felt simultaneous/dual interventions would be beneficial (if possibly optional), due to strong associations between smoking and other substances. Elements of the organizational and SUD community culture were identified as barriers. Treatment providers' also felt they lacked training and resources to support smoking cessation. They were further concerned about the impact of smoking cessation on mental health outcomes (e.g., negative affect) among clients.(16)

A qualitative study conducted with a national sample of 24 directors of SUD treatment programs (i.e., outpatient, residential, and methadone clinics) also revealed several barriers to implementing tobaccorelated policies and integrating tobacco cessation services within programs.(17) The directors noted that a traditional lack of focus on smoking cessation services within SUD treatment, client resistance, lack of financial support and resources, staff smoking rates, and environmental factors all served as barriers. These barriers hold potential to complicate successful integration of smoking cessation services into SUD treatment programs.

Further exploration of the barriers and facilitators, which may impact effective implementation, is key for successful integration. Often in SUD treatment programs, organizational factors that impact implementation remain unnoticed, despite evidence that they can be detrimental.(18) The Consolidated Framework for Implementation Research (CFIR) is a relatively new framework that synthesizes 18 existing implementation theories and evidence-based factors into a single taxonomy.(19)

The CFIR model includes five domains: (i) the intervention characteristics (ii) the outer setting, (iii) the inner setting, (iv) the characteristics of the individuals involved, and (v) the process of implementation. Thirty-nine constructs are then organized within these five domains, all of which interact with one another to impact (either positively or negatively) implementation. The model can be used to evaluate implementation, explain research findings, or assess context prior to implementation.(19) A systematic review of the CFIR model revealed that the model has been used in a broad way throughout the literature with interventions spanning several different topics, including mental health and physical health conditions.(20) Few studies employed the CFIR model to explore factors at the pre-implementation stage, though those studies that did were able to identify and address factors that could potentially impact implementation.(21-23)

Past research has shown treatment programs such as inpatient and residential, are more likely to adopt and sustain tobacco cessation interventions.(24) Thus, gaining a better understanding of these programs prior to implementation of a tobacco-related policy could serve as a method for facilitating the integration of tobacco cessation services. Using CFIR, this study aimed to identify key factors that could impact the 
implementation of tobacco cessation policies and services prior to integration in residential SUD treatment facilities.

\section{Methods}

\section{Program Selection and Recruitment}

This study examined data collected from three larger studies of residential SUD treatment programs described in more detail elsewhere.(25) All selected programs submitted application demonstrating an organizational aim to increase the capacity of their residential programs to offer tobacco cessation services and implement tobacco-free policies and received program level grant funding to accomplish the goal.(15) Across the three studies, 16 programs were enrolled. The 16 programs were located in 11 of California's 58 counties, from Lake County in the north to San Diego County in the south, spanning a distance of over 500 miles. At the time of participation, two programs had implemented tobacco free grounds with client-quit mandates. Data collection occurred in 2019.

\section{Data collection, procedures, and measures}

Using a purposeful sampling approach, 16 SUD residential program directors completed key-informant interviews between January and December 2019 during the pre-implementation stage of their respective smoking cessation intervention. Interviews were conducted by Zoom videoconferencing and lasted approximately 60 minutes. The interview covered topics within five CFIR domains as they related to tobacco policies and the integration of smoking cessation into SUD treatment. The interview guide is available as a supplemental material.

Directors also completed an online survey regarding director demographics and organizational characteristics. Demographic questions asked about race/ethnicity, gender, age, years of services in SUD treatment, and personal SUD recovery status. Organizational characteristics included 20 items drawn from prior research concerning tobacco free grounds, smoking among staff, and staff and clients smoking together.(25) The survey can be accessed at https://doi.org/10.6084/m9.figshare.11844975.v1 . Seven of the interviews were conducted by two of the authors (CMa and ES) and other research staff conducted the rest. All interviewers were female with moderate to expert skills in qualitative interviewing. Interviews were digitally recorded and transcribed. Interviewees received a \$50 gift card for their time. All participant information was de-identified to ensure confidentiality. Research procedures were approved by the Institutional Review Board of the University of California San Francisco.

\section{Qualitative Coding and Data Analysis}

Interviews were digitally recorded, professionally transcribed, and integrated into ATLAS.ti, a qualitative data management software program. Thematic analysis of interview transcripts was informed by grounded theory and guided deductively by the CFIR model. $(26,27)$ 
Members of the research team (KF, CMc, JW, CMa and ES) initially each read four transcripts independently identifying preliminary codes and subthemes. The first author then read all transcripts and, informed by the existing literature and the preliminary analysis of interviews, developed the initial codebook. Researchers met weekly over three months to compare preliminary coding choices, suggest possible codes and provide code definitions for the codebook. Differences between the coders were resolved by team discussion. Major themes were then mapped onto the domains and constructs of the CFIR. The transcripts were coded utilizing ATLAS.ti software. Two members of the research team independently coded a random sample of $20 \%$ of transcripts, which they had not previously coded, to establish $81 \%$ inter-rater agreement on parent (i.e., domain) codes. Finally, the first author selected passages that exemplified the themes, which mapped onto the CFIR constructs and domains.

Members of the coding team were also part of the larger research teams and therefore worked extensively with several of the agency directors represented in this sample. Therefore, the members of the coding team had an understanding of the workflow and current policies at several of the agencies and could therefore speak to the quality of the data.

\section{Results}

\section{Participants and Program Characteristics}

The participants were mostly female, with an average of over ten years of experience within the SUD treatment industry (Table 1 ). 
Table 1

Demographic Characteristics of Program Directors

\begin{tabular}{|ll|}
\hline & $\mathbf{n}(\%)$ \\
& $\mathbf{n = 1 6}$ \\
\hline Age (mean, SD)* & $48.9(8.5)$ \\
\hline Male & $6(37.5 \%)$ \\
\hline Race/Ethnicity* & \\
\hline Non-Hispanic White & $11(73.3 \%)$ \\
\hline Non-Hispanic Black/African American & $1(6.7 \%)$ \\
\hline Hispanic/Latino & $3(20.0 \%)$ \\
\hline Education* & \\
\hline Some college, associates, or professional license & $6(40 \%)$ \\
\hline Bachelor's & $1(6.7 \%)$ \\
\hline Masters & $8(53.3 \%)$ \\
\hline In recovery from substance use* & $7(46.7 \%)$ \\
\hline Smoking status** & \\
\hline Current smoker & $1(7.1 \%)$ \\
\hline Former smoker & $7(50.0 \%)$ \\
\hline Never smoker & $6(42.9 \%)$ \\
\hline Years working at agency & $4(25.0)$ \\
\hline Less than 1 year & $10(62.5 \%)$ \\
\hline $1-10$ years & \\
\hline Over 10 years & \\
\hline *missing $n=1, * *$ missing $n=2$ & \\
\hline
\end{tabular}

Half of the directors identified as persons in recovery from SUD. Program characteristics showed over half of this sample (75\%) allowed their clients to smoke nicotine products outdoors, and some programs (44\%) allowed clients to smoke during designated smoking breaks on campus or on off-campus walks. Half of the sample (50\%) allowed staff to smoke nicotine products outdoors, and a few programs (25\%) permitted their staff and clients to smoke together. See Table 2 for program policy features. 
Table 2

Program Policy Features

\begin{tabular}{|c|c|c|c|c|c|c|}
\hline $\begin{array}{l}\text { Program } \\
\#\end{array}$ & $\begin{array}{l}\text { Outdoor } \\
\text { smoking } \\
\text { allowed } \\
\text { for } \\
\text { clients? }\end{array}$ & $\begin{array}{l}\text { Outdoor } \\
\text { smoking } \\
\text { for } \\
\text { staff? }\end{array}$ & $\begin{array}{l}\text { Staff/clients } \\
\text { smoke } \\
\text { together }\end{array}$ & $\begin{array}{l}\text { Designated } \\
\text { smoking } \\
\text { breaks for } \\
\text { clients }\end{array}$ & $\begin{array}{l}\text { Any } \\
\text { NRT/cessation } \\
\text { medication } \\
\text { available } \\
\text { on/off } \\
\text { campus }\end{array}$ & $\begin{array}{l}\text { Smoking } \\
\text { cessation } \\
\text { screening or } \\
\text { any } \\
\text { counseling } \\
\text { available }\end{array}$ \\
\hline 1. & Yes & Yes & Yes & Unsure & No & Yes \\
\hline 2. * & No & No & No & No & Yes & Yes \\
\hline 3. & Yes & Yes & Yes & Yes & No & No \\
\hline 4. * & No & No & No & No & Yes & Yes \\
\hline 5. & Yes & No & No & No & Yes & Yes \\
\hline 6. & Yes & No & No & Yes & Yes & Yes \\
\hline 7. & No & No & Unsure & No & No & Yes \\
\hline 8. & Yes & Yes & No & Yes & No & Yes \\
\hline 9. & No & No & No & Yes & Yes & Yes \\
\hline 10. & Yes & No & No & No & Yes & Yes \\
\hline 11. & Yes & Yes & Yes & No & Yes & Yes \\
\hline 12. & Yes & Yes & No & Yes & Yes & Yes \\
\hline 13. & Yes & Yes & Yes & Yes & Yes & Yes \\
\hline 14. & Yes & Yes & No & No & No & Yes \\
\hline 15. & Yes & Yes & No & No & No & No \\
\hline 16. & Yes & No & No & Yes & Yes & Yes \\
\hline
\end{tabular}

We present findings within each of the five CFIR domains evaluated. Domains are in bold, CFIR subconstructs are in italics, and themes are underlined. Example quotes exemplifying each theme are displayed in Table 3.

\section{Domain I: Intervention Characteristics}

Intervention characteristics include key aspects of the intervention that could influence successful implementation.(19) Within the current study, the following findings emerged. 
Relative advantage. Participants expressed an interest in developing and implementing smoking cessation policies and services within their residential treatment program. They saw the potential reduction in smoking rates among clients through organizational-level interventions as an advantage over the current practice. Evidence strength and quality. There was uncertainty about whether residential treatment centers should permit e-cigarettes use (which have mixed evidence of effectiveness for tobacco product cessation).(28) Some directors perceived e-cigarettes as a tool to help smokers quit combustible tobacco products. These perceptions were reflected in organizational-level policies, which encouraged the use of e-cigarettes among clients and staff.

\section{Domain II: Outer setting}

The outer setting includes the larger context (e.g., political, social, economic) in which the organization resides.(19) Themes related to the outer are identified below. Needs and resources of those served by the intervention. All the participants described a degree of concern about residents' reaction to tobacco free grounds policies. Some directors believed clients did not have an interest in smoking cessation. Directors highlighted the prominent role that smoking can play within SUD recovery culture. According to directors, the SUD recovery culture often times prohibits the use of tobacco products to help ease the cessation of other substances. Directors further raised the concern with the organization culture, that prohibiting smoking in residential settings would change rapport between staff and clients. Directors therefore expressed some ambivalence toward removing smoking from residential treatment programs.

Directors of the two programs which had previously adopted and sustained tobacco-free policies and tobacco related services discussed their anticipated fears related to enforcing quit mandates, particularly as it related to client and staff resistance. However, they both reported that culture change was easier than they had initially anticipated. However, culture change was reportedly more challenging for other programs. Four directors described previous attempts to implement tobacco-free grounds that resulted in clients voluntarily leaving and the dismissal of clients for violation of the policy. According to one director, implementation of tobacco-free policies was a challenge, partially due to client's use of tobacco to cope

with comorbid mental health disorders. An additional challenge was the added workplace burden on staff (who may themselves be smokers) to ensure clients adhere to the policy. Negative consequences also included clients smoking tobacco in high fire risk places (e.g., in their bedroom or bathrooms). Within ten months of implementation, the policy was amended to permit designated smoke breaks for clients. External policy and incentives. Many directors were aware of current government mandates (e.g., city, county, state, federal) related to nicotine products. However, they reported their programs did not enforce such mandates. For example, in 2018, San Francisco County passed the ban of flavored tobacco products including menthol products. $(29,30)$ Program directors remarked they ensured their clients followed public no-smoking rules when participating in program activities off campus (e.g., no smoking in public areas when going on a walk).

Directors reported external incentives such as grant funding, were important factors in their motivation to integrate tobacco cessation services in SUD treatment. Some directors stated they were able to provide 
nicotine replacement options and smoking cessation services to their clients through partial funding from grants or private donations.

\section{Domain III: Inner setting}

The inner setting includes factors of the program (e.g., structural, cultural contexts) that may be associated with implementation.(19) The following themes emerged related to the inner setting. Implementation climate. Residential directors believed that the implementation climate of their programs was compatible with smoking cessation interventions and, tobacco-related services were a priority in SUD treatment. Directors believed that smoking cessation was a priority because of the impact of smoking on their clients' overall health. They reported using a holistic approach to providing SUD treatment, and acknowledged the health risks associated with smoking. Some directors demonstrated strong leadership commitment to enforce an institutional smoking ban. Readiness for implementation. Despite directors' expressed strong commitment to develop and implement smoking cessation policies, they also noted major reasons why they had not offered treatment for clients including: the lack of available resources such as, workforce expertise and therapeutic interventions (pharmacotherapy and psychotherapy), financial reimbursement and SUD counselors smoking status. Directors reported low staff training/knowledge about tobacco-related services. Workforce resources are further discussed under the CFIR construct, Planning and Engaging. Most programs screened clients for nicotine use disorders and some occasionally provided informal smoking cessation counseling as part of wellness process groups. However, several directors mentioned that smoking cessation counseling services are not reimbursed under the current public financial reimbursement system. Although clients may buy forms of nicotine replacement treatment (NRT) over-the-counter, directors acknowledged the cost of smoking cessation medications as a barrier for clients. Therefore, programs sought public health grant funding to subsidize NRT for clients unable to pay. Programs unable to obtain funding used a referral to NRT approach, by referring residents to local clinics and national quit lines. Directors recognized workflow as a viable concern in the implementation process. Directors noted that the structure of their programs do not have workflow processes and services such as onsite healthcare services that would allow medical personnel to prescribe and dispense NRT.

With respect to staff smoking status, most programs did not require staff to be nicotine-free nor did they provide smoking cessation services for staff. Directors expressed a desire to aid employees in achieving better health outcomes (e.g., refer to an EAP) but did not find it appropriate to impose smoking cessation mandates for their employees.

Directors reported that they anticipated state policy makers would eventually impose smoking-free campus mandates. Thus, many expressed being highly motivated to participate in this research study simply because the project provided access to smoking cessation services and training. Others expressed the need for standardized or uniform tobacco cessation policies across all facilities providing SUD treatment services.

\section{Domain IV: Characteristics of Individuals}


Characteristics of individuals in an organization includes factors of the individual's beliefs, knowledge, self-efficacy, and personal attributes that may be associated with implementation.(19) Self-efficacy. Despite the challenges of service and policy integration, all directors expressed self-efficacy and optimism that they could successfully incorporate smoking cessation interventions into SUD treatment curricula. They all reported a strong commitment to integrating smoking cessation interventions within their treatment programs and acknowledged the potential health benefits for clients and staff.

\section{Domain V: Process of implementation}

The process of implementation includes stages of implementation such as planning, executing, reflecting and evaluating, and the presence of key intervention stakeholders and influencers including opinion leaders, stakeholder engagement, and project champions.(19) The following theme emerged related to the process of implementation. Planning and engaging. Directors conducted planning activities that included assessing their environmental settings, and workforce knowledge to identify potential barriers to implementation. Directors also recognized the need for client champions and stakeholders.

SUD residential treatment directors discussed that executive committees developed policies, most often without client input, which were communicated to residential clients via a meeting (e.g., "a house meeting"). Clients' reactions to policies served as a catalyst for policy amendments that had occurred in some programs. Some directors therefore suggested that residents should be engaged in the process of developing policies and services, while another suggested that the implementation approach should be gradual and repetitive.

\section{Discussion}

This study applied the CFIR framework to identify barriers and facilitators that influenced the development of tobacco policies and services in residential SUD treatment programs. All five CFIR domains emerged from the analysis: intervention characteristics, outer setting, inner setting, characteristics of individuals, and the process of implementation. However, the outer setting and inner setting domains were represented most prominently in the data both as barriers and facilitators at preimplementation. Leadership engagement and commitment to adopting tobacco cessation polices and services were key facilitators. Across all participating programs, directors were highly engaged, motivated, and had self-efficacy to implement tobacco cessation policies and services. Integrating smoking cessation polices and services in residential SUD treatment programs was viewed as compatible with a holistic approach to the treatment of SUDs, superior to current practices, and facilitated by local government mandates and incentives. However, important barriers to implementation were identified within several CFIR domains: the intervention characteristics, outer setting, inner setting, and process of implementation.

In considering the inner setting, directors felt that existing resources were insufficient to support implementation of comprehensive smoking cessation policies. Program directors cited a number of barriers related to the inner setting that impacted the extent to which they could change policies, services, 
and practices including the resources available to treat tobacco use disorder, financial costs of NRT medications, and the ability to be reimbursed for smoking cessation services. They also cited the SUD recovery culture as an important barrier to adopting tobacco cessation polices in SUD treatment. According to directors the SUD recovery culture was connected to both staff and client resistance to tobacco-free grounds. They further explained the organizational culture that permitting clients to smoke on the premises was used to facilitate prosocial behaviors and that smoking played an important role in helping clients to cope with stress and build provider rapport. Other barriers specific to the inner setting centered on the organizational culture: ambivalence about imposing tobacco-free grounds, uneven attention given to staff smoking, and the view that promoting smoking cessation among staff was not part of their role. Prior research has documented these barriers to the integration of smoking cessation care within SUD treatment programs. $(17,31-34)$ In SUD residential treatment, rapport is a critical skill and clients perceive supportive staff as motivators for their personal tobacco cessation efforts. (16)

In our study, directors discussed their reluctance to address smoking among their workforce. However, to increase the success of tobacco cessation policy interventions, it is critical to address staff smoking. Staff smoking is common in SUD treatment and could reinforce client tobacco use.(4) In a recent study, Guydish and colleagues found that higher rates of SUD treatment program staff and clients smoking together was associated with lower rates of client intent to quit smoking in the next 30 days, more negative client attitudes toward quitting smoking, and with clients receiving fewer tobacco services.(35) This finding highlights the importance of addressing staff smoking in SUD treatment programs and provides evidence for a policy to prohibit staff smoking together with clients.

The successful implementation of tobacco services in residential SUD treatment centers would require organizational culture change interventions. Interventions would include program wide staff and client training and workshops on the long term effectiveness of tobacco cessation services,(36) policies to prohibit staff and clients from smoking together $(31,35)$ and, provide holistic avenues for staff to build rapport with clients, such as gardening or sports. Organizational change interventions have been associated with increased favorable attitudes toward treating tobacco use disorder in SUD treatment programs, use of NRT medications, client receipt of services from their programs or counselors, and a reduction client smoking prevalence and cigarettes smoked per day. $(36,37)$

Furthermore, funding streams continue to serve as a barrier to the availability of smoking cessation services in SUD treatment programs.(34) Smoking cessation covered services have expanded with the expansion of Medicaid under the Affordable Care Act (ACA). In our sample the state of California, MediCal, currently cover SUD treatment and tobacco-related cessation counseling and medication for clients receiving outpatient and inpatient hospitalization services. Presently, residential SUD treatment programs are not Medi-Cal-recognized practitioners of tobacco related cessation services and SUD providers' services are not reimbursed in residential settings. In California, the Department of Health Care Services (DHCS) has sole authority to license nonmedical SUD treatment facilities does not consider tobaccorelated services within its scope. Therefore, SUD program directors have no regulatory or financial incentive to engage in tobacco-related cessation services. Unfortunately, this gap in clinical care has a 
negative impact on the prevalence of smoking among residential clients and its associated health comorbidities.(38) Clients are more likely to have a quit smoking when they have health coverage, and are three times more likely to quit smoking while they are in SUD treatment.(39) Therefore, there is a critical need for the allocation of resources dedicated to build SUD programs' capacity to provide smoking cessation services including expanded insurance coverage of counseling-based smoking cessation programs.

Directors reported implementing changes to the program's smoking polices using a top-down approach where client input or participation in policymaking was not taken into consideration. Engaging clients as stakeholders early in the design of interventions is essential for implementation success.(40) Clients could provide an insider perspective on the acceptability of specific policies or intervention components that could enhance buy-in. Furthermore, implementation will be more effective when all key stakeholders are involved (e.g., leadership, external change agents, clients).(41) A communication strategy should be in place before implementation to educate both providers and clients about the value of tobacco-free grounds and smoking cessation services.

\section{Limitations}

Certain limitations of the study should be noted. The study focused on SUD residential treatment programs in California, and further research is needed to understand how these findings may apply in other settings. The factors influencing adoption of tobacco cessation polices and services may be different in outpatient settings. Our analysis relies on self-report from program directors, and thus does not take into account the views of clients or other staff members. Obtaining the perspective of other key stakeholders would increase our understanding about barriers and solutions from multiple perspectives. We did not include data collection during or following implementation of an intervention, thus director perceptions about the implementation process are not represented. Finally, the residential treatment programs that participated in this study responded to a call for applications for SUD treatment programs willing to participate in implementing tobacco policy change interventions. Thus, residential programs that had an investment in and a higher level of motivation to participate in this study may be overrepresented.

\section{Conclusions}

The CFIR provided a useful framework for understanding the many influences at play when implementing tobacco policies and services into a complex setting such as residential addiction treatment. Study findings indicate that challenges related to reimbursement for smoking cessation services persist in the context of SUD treatment programs. To increase adoption of tobacco policies and services in SUD treatment settings, it is essential to dedicate funding to increase training capacity, but also to expand reimbursement of smoking cessation-counseling services in SUD treatment.

\section{Abbreviations}


CFIR: Consolidated Framework for Implementation Research

CTCP: California Tobacco Control Program

DHCS: Department of Health Care Services

NRT: Nicotine replacement treatment

SUD: Substance use disorders

\section{Declarations}

\section{Ethics approval and consent to participate:}

The Institutional Review Board of the University of California San Francisco approved this manuscript's research procedures.

\section{Consent for publication:}

Not applicable.

\section{Availability of data and materials:}

The datasets used and analyzed during the current study are available from the corresponding author on reasonable request. We confirm we had full access to all of the data in this study and we take responsibility for the integrity of the data and the accuracy of the data analysis.

\section{Competing interests:}

We wish to confirm that there are no known conflicts of interest associated with this publication and there has been no significant financial support for this work that could have influenced its outcome. We confirm that there are no known potential competing interests.

\section{Funding:}

This work was supported by the California Tobacco Control Program (18-10025), by the California Tobacco Related Disease Research Program (TRDRP 27IR-0040, 28CP-0038), and by the National Institute on Drug Abuse, Award Number T32DA007250. The content is solely the responsibility of the authors and does not necessarily represent the official views of the National Institutes of Health. The abovementioned funding sources played no role in the design of the study, data collection, analysis, and interpretation of data; and they did not play a role in writing the manuscript.

\section{Authors' contributions:}


We confirm that the manuscript has been read and approved by all named authors and that there are no other persons who satisfied the criteria for authorship but are not listed. We confirm that this publication is not under review-and will not be under review-by another publication while it is being considered by Substance Abuse Treatment, Prevention, and Policy. JKF contributed to the conception of this manuscript's research hypothesis, design, analysis, interpretation, drafting and revision of the manuscript. $\mathrm{CMc}$ contributed to the design, analysis, data interpretation, drafting and revision of the manuscript. CMa contributed to the design, analysis, data interpretation, drafting and revision of the manuscript. VG contributed to the data analysis, data interpretation and revision of the manuscript. ES, and JW contributed to data collection, data analysis and provided revisions to the manuscript. JG contributed to study design, and revision of the manuscript. All authors provided critical feedback and helped shape the research, analysis and manuscript.

\section{Acknowledgements:}

Not applicable

\section{References}

1. Weinberger AH, Gbedemah M, Wall MM, Hasin DS, Zvolensky MJ, Goodwin RD. Cigarette use is increasing among people with illicit substance use disorders in the United States, 2002-14: emerging disparities in vulnerable populations. Addiction. 2018;113(4):719-28.

2. Guydish J, Passalacqua E, Pagano A, Martínez C, Le T, Chun J, et al. An international systematic review of smoking prevalence in addiction treatment. Addiction. 2016;111(2):220-30.

3. Bandiera FC, Anteneh B, Le T, Delucchi K, Guydish J. Tobacco-Related Mortality among Persons with Mental Health and Substance Abuse Problems. PLOS ONE. 2015 Mar 25;10(3):e0120581.

4. Baca CT, Yahne CE. Smoking cessation during substance abuse treatment: What you need to know. J Subst Abuse Treat. 2009;36(2):205-19.

5. Weinberger AH, Platt J, Esan H, Galea S, Erlich D, Goodwin RD. Cigarette smoking is associated with increased risk of substance use disorder relapse: A nationally representative, prospective longitudinal investigation. J Clin Psychiatry. 2017 Feb;78(2):e152-60.

6. Conrad M, Bolte T, Gaines L, Avery Z, Bodie L. The Untreated Addiction: Going Tobacco-Free in a VA Substance Abuse Residential Rehabilitation Treatment Program (SARRTP). J Behav Health Serv Res. 2018 Oct 1;45(4):659-67.

7. Asamsama OH, Miller SC, Silvestri MM, Bonanno C, Krondilou K. Impact of implementing a tobacco and recreational nicotine-free policy and enhanced treatments on programmatic and patient-level outcomes within a residential substance use disorder treatment program. J Subst Abuse Treat. 2019 Dec 1;107:44-9.

8. Richey R, Garver-Apgar C, Martin L, Morris C, Morris C. Tobacco-Free Policy Outcomes for an Inpatient Substance Abuse Treatment Center. Health Promot Pract. 2017 Jul 1;18(4):554-60. 
9. Romano I, Costello MJ, Ropp C, Li Y, Sousa S, Bruce D, et al. Evaluating the short-term impact of a tobacco-free policy in an inpatient addiction treatment setting. J Subst Abuse Treat. 2019 Dec $1 ; 107: 50-9$.

10. Brown E, Nonnemaker J, Federman EB, Farrelly M, Kipnis S. Implementation of a tobacco-free regulation in substance use disorder treatment facilities. J Subst Abuse Treat. 2012 Apr 1;42(3):31927.

11. Correa-Fernández V, Wilson WT, Kyburz B, O’Connor DP, Stacey T, Williams T, et al. Evaluation of the Taking Texas Tobacco Free Workplace Program within behavioral health centers. Transl Behav Med. 2019 Mar 1;9(2):319-27.

12. Drach LL. Promoting Smoke-free Environments and Tobacco Cessation in Residential Treatment Facilities for Mental Health and Substance Addictions, Oregon, 2010. Prev Chronic Dis [Internet]. 2012 [cited 2021 Feb 1];9. Available from: https://www.cdc.gov/pcd/issues/2012/11_0080.htm

13. Marshall LL. Strategies to Support Tobacco Cessation and Tobacco-Free Environments in Mental Health and Substance Abuse Facilities. Prev Chronic Dis [Internet]. 2015 [cited 2021 Feb 1];12. Available from: https://www.cdc.gov/pcd/issues/2015/14_0585.htm

14. Williams JM, Foulds J, Dwyer M, Order-Connors B, Springer M, Gadde P, et al. The integration of tobacco dependence treatment and tobacco-free standards into residential addictions treatment in New Jersey. J Subst Abuse Treat. 2005 Jun 1;28(4):331-40.

15. CTCP. CG 18-10137 Initiative to Reduce Tobacco-Related Disparities at Residential Behavioral Health Facilities [Internet]. 2018. Available from: https://tcfor.catcp.org/index.cfm? fuseaction=opportunities.viewOpp\&oppID=63.

16. Gentry S, Craig J, Holland R, Notley C. Smoking cessation for substance misusers: A systematic review of qualitative studies on participant and provider beliefs and perceptions. Drug Alcohol Depend. 2017 Nov 1;180:178-92.

17. Pagano A, Tajima B, Guydish J. Barriers and Facilitators to Tobacco Cessation in a Nationwide Sample of Addiction Treatment Programs. J Subst Abuse Treat. 2016 Aug 1;67:22-9.

18. Damschroder LJ, Hagedorn HJ. A guiding framework and approach for implementation research in substance use disorders treatment. Psychol Addict Behav J Soc Psychol Addict Behav. 2011 Jun;25(2):194-205.

19. Damschroder LJ, Aron DC, Keith RE, Kirsh SR, Alexander JA, Lowery JC. Fostering implementation of health services research findings into practice: a consolidated framework for advancing implementation science. Implement Sci. 2009 Aug 7;4(1):50.

20. Kirk MA, Kelley C, Yankey N, Birken SA, Abadie B, Damschroder L. A systematic review of the use of the Consolidated Framework for Implementation Research. Implement Sci. 2016 May 17;11(1):72.

21. English M. Designing a theory-informed, contextually appropriate intervention strategy to improve delivery of paediatric services in Kenyan hospitals. Implement Sci. 2013 Mar 28;8(1):39.

22. Robins LS, Jackson JE, Green BB, Korngiebel D, Force RW, Baldwin L-M. Barriers and Facilitators to Evidence-based Blood Pressure Control in Community Practice. J Am Board Fam Med. 2013 Sep 
$1 ; 26(5): 539-57$.

23. VanDevanter N, Kumar P, Nguyen N, Nguyen L, Nguyen T, Stillman F, et al. Application of the Consolidated Framework for Implementation Research to assess factors that may influence implementation of tobacco use treatment guidelines in the Viet Nam public health care delivery system. Implement Sci. 2017 Dec;12(1):1-8.

24. Eby LT, Laschober TC, Muilenburg JL. Sustained, New, Never, and Discontinued Tobacco Cessation Services Adopters. J Subst Abuse Treat. 2015 Feb;0:8-14.

25. Guydish J, Kapiteni K, Le T, Campbell B, Pinsker E, Delucchi K. Tobacco use and tobacco services in California substance use treatment programs. Drug Alcohol Depend. 2020 Sep 1;214:108173.

26. Boyatzis RE. Transforming qualitative information: Thematic analysis and code development. Thousand Oaks, CA, US: Sage Publications, Inc; 1998. xvi, 184 p. (Transforming qualitative information: Thematic analysis and code development).

27. Glaser BG, Strauss AL. The discovery of grounded theory: strategies for qualitative research. 4. paperback printing. New Brunswick: Aldine; 2009. 271 p.

28. Kalkhoran S, Glantz SA. E-cigarettes and smoking cessation in real-world and clinical settings: a systematic review and meta-analysis. Lancet Respir Med. 2016 Feb;4(2):116-28.

29. Yang YT, Glantz S. San Francisco Voters End the Sale of Flavored Tobacco Products Despite Strong Industry Opposition. Ann Intern Med. 2018 Nov 20;169(10):708-9.

30. Vyas P, Ling P, Gordon B, Callewaert J, Dang A, Smith D, et al. Compliance with San Francisco's flavoured tobacco sales prohibition. Tob Control. 2021 Mar;30(2):227-30.

31. Guydish J, Passalacqua E, Tajima B, Manser ST. Staff Smoking and Other Barriers to Nicotine Dependence Intervention in Addiction Treatment Settings: A Review. J Psychoactive Drugs. 2007 Dec;39(4):423-33.

32. Laschober TC, Muilenburg JL, Eby LT. Factors Linked to Substance Use Disorder Counselors' (Non)Implementation Likelihood of Tobacco Cessation 5 A's, Counseling, and Pharmacotherapy. J Addict Behav Ther Rehabil [Internet]. 2015 [cited 2021 Feb 4];4(1). Available from: https://www.ncbi.nlm.nih.gov/pmc/articles/PMC4436588/

33. Fallin-Bennett A, Parker KA, Miller A, Ashford K, Hahn EJ. Smoking and Tobacco-Free Policies in Women's Residential Substance Use Disorder Treatment Facilities: A Community-Engaged Approach. Nicotine Tob Res. 2018 Sep 25;20(11):1386-92.

34. Knudsen HK. Implementation of smoking cessation treatment in substance use disorder treatment settings: a review. Am J Drug Alcohol Abuse. 2017 Mar 4;43(2):215-25.

35. Guydish J, Le T, Campbell B, Yip D, Ji S, Delucchi K. Drug abuse staff and clients smoking together: A shared addiction. J Subst Abuse Treat. 2017 May 1;76:64-8.

36. Guydish J, Ziedonis D, Tajima B, Seward G, Passalacqua E, Chan M, et al. Addressing Tobacco Through Organizational Change (ATTOC) in residential addiction treatment settings. Drug Alcohol Depend. 2012 Feb 1;121(1-2):30-7. 
37. Guillaumier A, Skelton E, Shakeshaft A, Farrell M, Tzelepis F, Walsberger S, et al. Effect of increasing the delivery of smoking cessation care in alcohol and other drug treatment centres: a clusterrandomized controlled trial. Fac Soc Sci - Pap Arch [Internet]. 2019 Jan 1; Available from: https://ro.uow.edu.au/sspapers/4659

38. Guydish J, Kapiteni K, Le T, Campbell B, Pinsker E, Delucchi K. Tobacco use and tobacco services in California substance use treatment programs. Drug Alcohol Depend. 2020 Sep 1;214:108173.

39. Yip D, Gubner N, Le T, Williams D, Delucchi K, Guydish J. Association of Medicaid Expansion and Health Insurance with Receipt of Smoking Cessation Services and Smoking Behaviors in Substance Use Disorder Treatment. J Behav Health Serv Res. 2020 Apr 1;47(2):264-74.

40. Pronovost PJ, Berenholtz SM, Needham DM. Translating evidence into practice: a model for large scale knowledge translation. BMJ. 2008 Oct 6;337:a1714.

41. Greenhalgh T, Robert G, Macfarlane F, Bate P, Kyriakidou O. Diffusion of Innovations in Service Organizations: Systematic Review and Recommendations. Milbank Q. 2004 Dec;82(4):581-629.

\section{Table}

Due to technical limitations, table 3 xlsx is only available as a download in the Supplemental Files section.

\section{Supplementary Files}

This is a list of supplementary files associated with this preprint. Click to download.

- SupplementalDirectorInterviewGuide.docx

- Table3summaryofqualitativethemesandsamplequotations.xlsx 